\title{
Highway Electric Vehicle Charging Load Prediction and its Impact on the Grid
}

\author{
Huiyi Wang ${ }^{a}$, Xueliang Huang ${ }^{b}$, Lixin Chen ${ }^{c}$ and Yuqi Zhou ${ }^{d}$ \\ School of Electrical Engineering, Southeast University, Nanjing 210096, China \\ awhy2932@gmail.com, bxlhuang@seu.edu.cn \\ cchenlixing19851210@163.com, dzhouyq813@163.com
}

\begin{abstract}
Keywords: highway, electric vehicles, load prediction, Floyd algorithm, Monte Carlo method, the impact on grid.

Abstract. In this passage, a new method is proposed to predict the load of electric vehicles (EVs) on highway. In this method, a simplified highway network containing charging stations is established, based on which we can generate an adjacency matrix. Then the origin and destination (OD) of vehicles are extracted according to the probability density function (PDF) built by the statistic of highway toll station. Floyd algorithm is used to determine the routine of EVs. Departure time, state of charge (SOC), battery capacity, driving speed and other parameters are all extracted according to PDF. Monte Carlo method is attached to simulate large-scale EV load on highway. Highway network of Jiangsu province is proposed as an example, and impact of EV load on power grid is evaluated by voltage deviation and loss.
\end{abstract}

\section{Introduction}

The number of plug-in electric vehicles is observing a significant increase in the recent years because of its strong potential to decrease oil consumption [1]. In this occasion, accurate load prediction of electric vehicle is important for the stability of power grid. EV load in city can be predicted by parking demand in residential and office buildings [2], charging model of different vehicles [3] or other methods. However, nowadays, with the technological improvement of electric vehicles and battery, long-distance travel of electric vehicles is possible, which means an increasing number of EV on highway. Large-scale electric vehicle on the highway will inevitably lead to load growth [4]. In this paper, we propose a method to predict the load profile of each charging station in highway, and estimate its impact on the power grid. Firstly, we set the model of single electric vehicle load and large-scale electric vehicle load; secondly, the network of highway with charging stations is established and simplified, in which Jiangsu province is used as an example; thirdly, we determine the number of electric vehicles varies with space profile using OD data, and determine the number of electric vehicles varies with time profile; lastly, Monte Carlo method is used to simulate, in which Floyd algorithm is attached to determine the route of each electric vehicle. Impact on power grid is calculated in IEEE 39 nodes.

\section{Single Electric Vehicle Modeling}

There are three main parameters in the model of single EV: departure time, state of charge and its routine in highway.

\section{Departure time}

Departure time of vehicles can be classified into two category: sedan car and freight car. Two picks can be observed in the profile of sedan car number changing through time in Fig.1, which means most people driving sedan cars choose to depart at 8:00-10:00am and 16:00-18:00pm during a day. In contrast, drivers of freight car prefer to depart at 10pm-8am, observed in Fig.2, to reduce traffic accidents. 


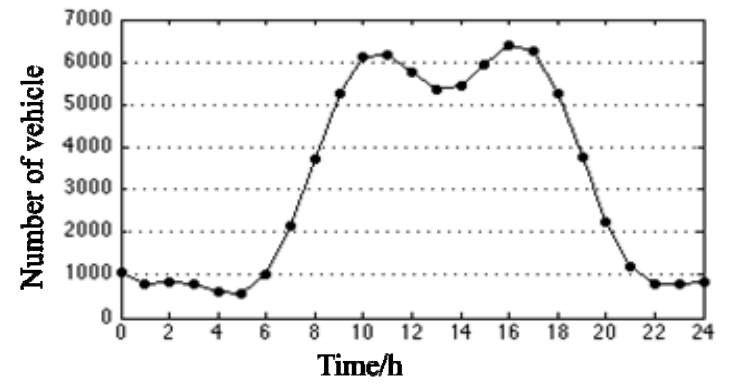

Fig.1 sedan car number-time profile

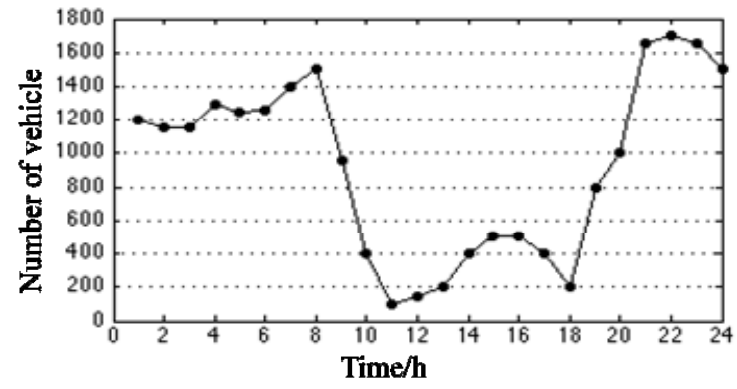

Fig.2 freight car number-time profile

We can calculate the probability function of departure time as follows:

$$
\begin{aligned}
& P_{S}=0.04-0.03 \cos (0.3 t)-0.03 \sin (0.3 t)+0.001 \cos (0.6 t)-0.006 \sin (0.6 t) \\
& -0.008 \cos (0.89 t)+0.0065 \sin (0.89 t) \\
& P_{f}=0.03 \cos (0.25 t)-0.007 \cos (0.25 t)-0.005 \cos (0.25 t)+0.006 \sin (0.25 t) \\
& -0.01 \sin (0.5 t)+0.007 \sin (0.25 t)-0.01 \sin (0.75 t)+0.04
\end{aligned}
$$

Where $P_{s}$ is the probability of sedan vehicle's departure time, and $P_{f}$ is the probability of freight vehicle's departure time.

\section{State of charge}

EV's state of charge express its remaining battery capacity. It declines along with time as soon as the vehicle start at a speed of $v_{s o c}(t)$, so that EV's state of charge at $t$ can expressed as:

$$
\begin{aligned}
& S(t)=S_{d}+\Delta S_{t}-\int_{t_{m}}^{t} v_{S O C}(t) d t \\
& \bar{v}_{S O C}=\bar{v} \frac{W_{100, k}}{100 Q_{k}}
\end{aligned}
$$

Where $t m$ is the departure time, $S_{d}$ is the SOC in departure time. If the vehicle charges before t, the change of SOC is $\Delta S t . W 100, k$ is the power consumption per $100 \mathrm{~km}$ and $Q_{k}$ is the battery capacity.

The charging process of EV contains two parameters: charging time and charging power. Actually, the charging process of EV is consisted of three periods as Fig.3. But in this passage, the charging power is simplified into a constant between $2-3 \mathrm{~kW}$.

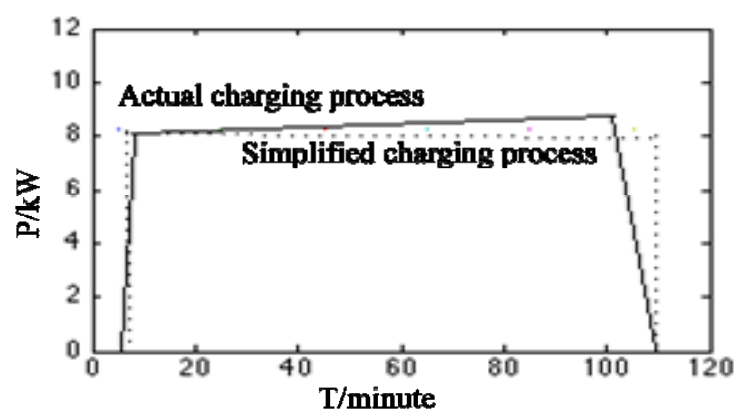

Fig.3 charging process of EV

The charging time of EV can be expressed as:

$$
T_{C}=\frac{\left(\alpha-S O C_{0}\right)}{\eta \times P_{c}} \times Q_{k} \times 60
$$

Where $P_{c}$ is the charging power, $S O C_{0}$ is the state of charge at the start of charging process. 


\section{The routine of $\mathrm{EV}$ determined by Floyd algorithm}

The routine of each EV is determined by its origin and destination and the highway network, using Floyd algorithm.

A pair of vertices $(i, j)$ is selected to find the shortest path. For each of these pairs of vertices, the true shortest path could be either: (1) a path that only uses vertices in the set $\{1 \ldots k\}$ or (2) a path that goes from $i$ to $k+1$ and then from $k+1$ to $j$. So we can express Floyd algorithm as follow:

$$
\operatorname{Stp}(i, j, k+1)=\min (\operatorname{Stp}(i, j, k), \operatorname{Stp}(i, k+1, k)+\operatorname{Stp}(k+1, j, k))
$$

The algorithm works by first computing all $(i, j)$ pairs for $k=1, k=2$, until $k=N$, until we have found the shortest path for all $(i, j)$ pairs using any intermediate vertices. We judge the shortest path as the routine that drivers will choose.

\section{Large-scale electric Vehicle Modeling}

\section{Simplified highway network}

A simplified highway network consists of origin, destination, charging station and routines. In this passage, we take Jiangsu province as an example, highway network can be simplified as Fig.4, in which nodes 1-24 are charging stations, and nodes 35-48 are OD cities.

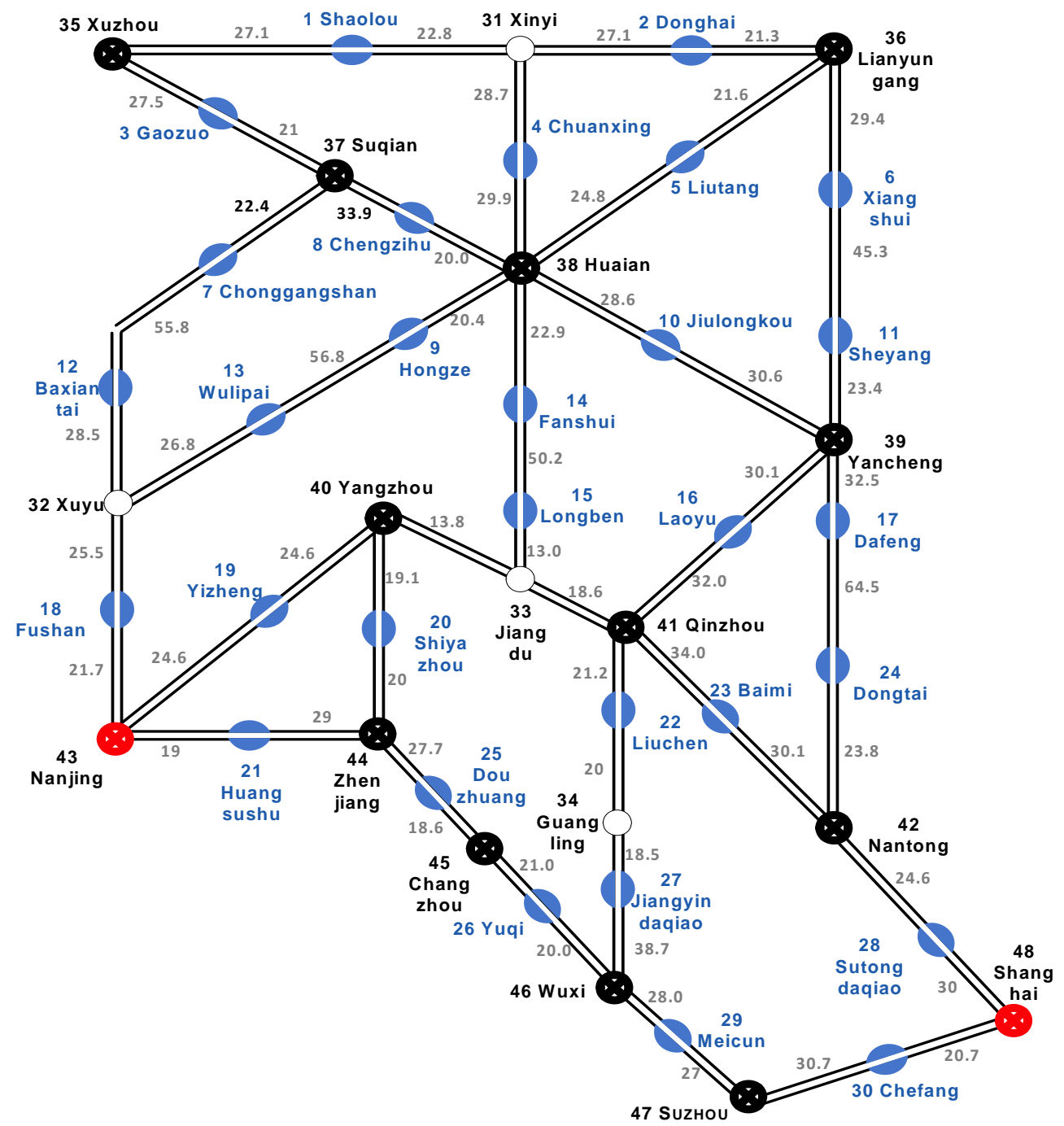

Fig.4 simplified highway network of Jiangsu

So we can build the Adjacency Matrix of the highway network of Jiangsu as follow: 


$$
A[i, j]=\left\{\begin{array}{c}
w_{i j},\left(v_{i}, v_{j}\right) \in E(G) \\
0 / \infty,\left(v_{i}, v_{j}\right) \notin E(G)
\end{array}\right.
$$

Where $w_{i j}$ is weights on edges, and $\left(v_{i} \cdot v_{j}\right)$ is the distance between node $v_{i}$ and node $v_{j}$.

\section{OD distribution}

To make Floyd algorithm function, the OD of each vehicle must be determined. Utilizing the statistic of toll gate, the distribution of OD can be expressed as Table.1:

Table.1 OD distribution

\begin{tabular}{cccccccc}
\hline & 1Nanjing & 2Wuxi & 3Xuzhou & 4Changzhou & 5Suzhou & 6Nantong & 7Lianyungang \\
\hline Po & 0.1133 & 0.1438 & 0.0665 & 0.0525 & 0.3349 & 0.0739 & 0.0363 \\
Pd & 0.1127 & 0.1475 & 0.0607 & 0.0508 & 0.3354 & 0.0725 & 0.0336 \\
\hline & 8Huai'an & 9Yancheng & 10Yangzhou & 11Zhenjiang & 12Taizhou & 13Suqian & \\
\hline Po & 0.0256 & 0.0308 & 0.0327 & 0.0224 & 0.0496 & 0.0178 & \\
Pd & 0.0243 & 0.0265 & 0.0410 & 0.0207 & 0.0572 & 0.0172 & \\
\hline
\end{tabular}

Where $P_{o}$ is the probability of departing from this city, and $P_{d}$ is the probability of going to this city. The table can also expressed by a distribution function as follow:

$$
N_{O}=\left\{\begin{array}{l}
1, x \in[0,1133] \\
2, x \in[1134,2571] \\
\cdots \\
13, x \in[9822,10000]
\end{array}, \quad N_{D}=\left\{\begin{array}{l}
1, x \in[0,1127] \\
2, x \in[1128,2603] \\
\cdots \\
13, x \in[9828,10000]
\end{array}\right.\right.
$$

Where $x$ is a uniformly distributed random variables in $[0,10000] . N$ is the city number expressed in Table.1.

\section{The simulation of EV Load}

\section{Sampling from Probability Distribution Functions}

We apply Acceptance-Rejection Algorithm to sample from probability distribution functions of departing time, SOC, etc. of EVs.

It is started by inputting probability density function $f(x)$, where $\mathrm{x}$ varies from [a, b] and the function has a bound as follow:

$$
\max \{f(x) \mid a \leq x \leq b\}=c
$$

Firstly, generating a uniformly distributed random number $x$ in the interval [a, b]; Secondly, generating a uniformly distributed random number $y$ in the interval $[0, \mathrm{c}]$. Thirdly, if $y \leq f(x)$, we accept $x$ as the required random number. Otherwise, returning to the first step to extract a new pair of $(\mathrm{x}, \mathrm{y})$. We extract $x$ at the beginning of cycle.

\section{Load prediction based on Monte Carlo method}

Monte Carlo methods can be used to solve any problem having a probabilistic interpretation. Usually, it tends to follow a particular pattern. Detailed flow chart is showed in Fig.4. Firstly, defining a domain of possible inputs. In this passage, possible inputs contains three constants: charging power, battery capacity and power consumption per $100 \mathrm{~km}$ of EV. And three parameters: departure time, driving speed and routine. Secondly, generate inputs randomly from a probability distribution over the domain. The methods is showed in 4.1. Thirdly, perform a deterministic 
computation on the inputs. In this passage, we compute 20000 vehicles. Lastly, aggregate the results. The simulation results are as Fig.5 and Fig.6.

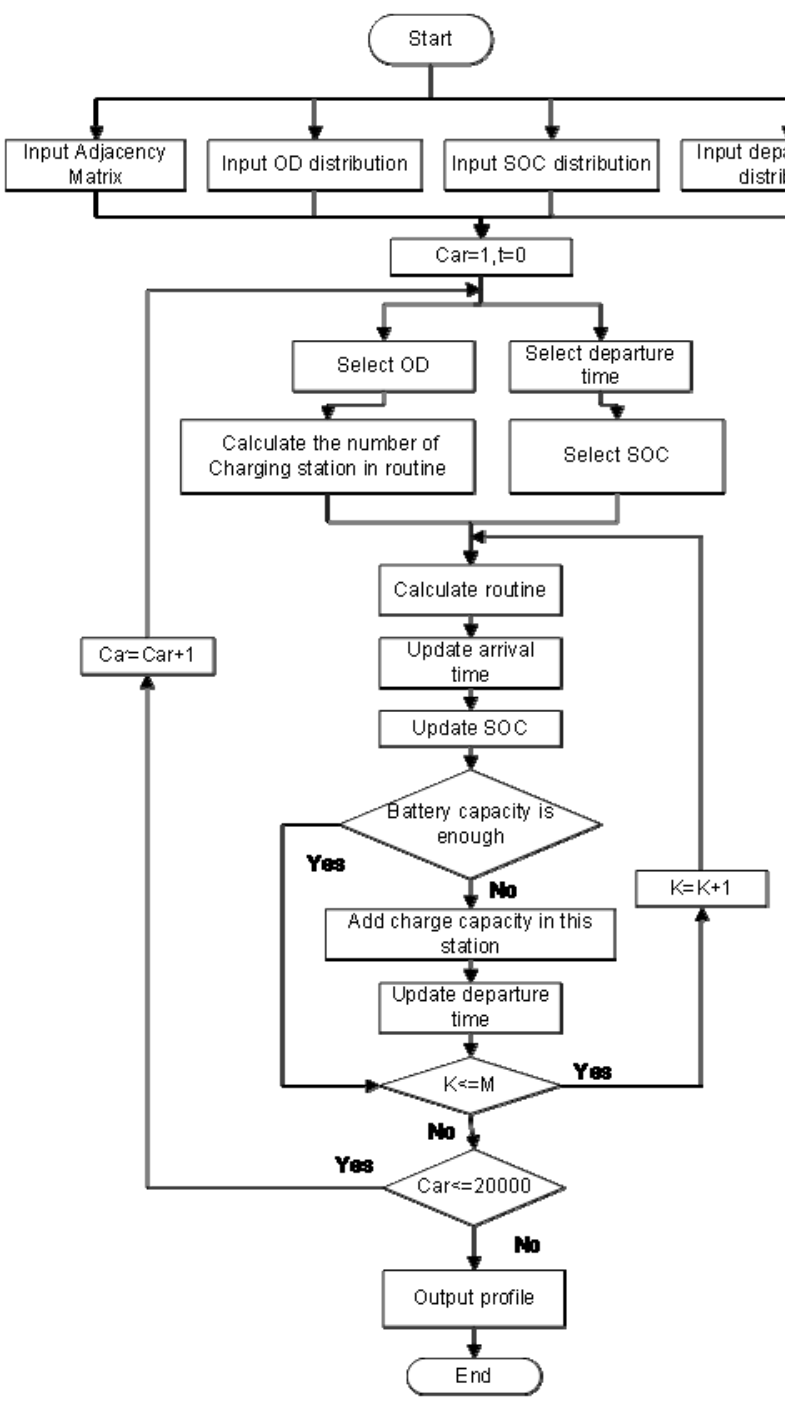

Fig.5 flow chart of simulation

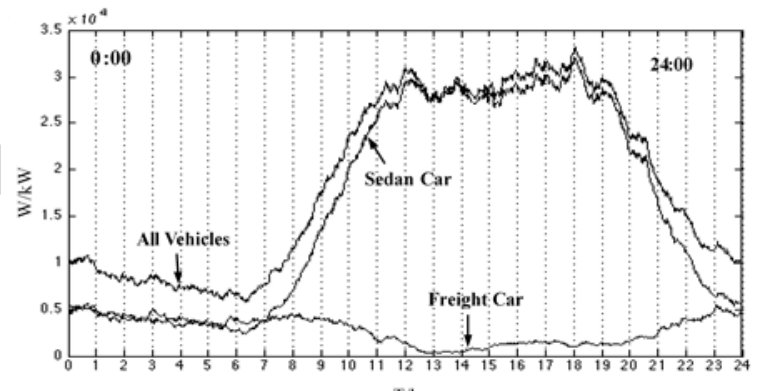

$\mathrm{T} / \mathrm{h}$

$\mathrm{T} / \mathrm{h}$
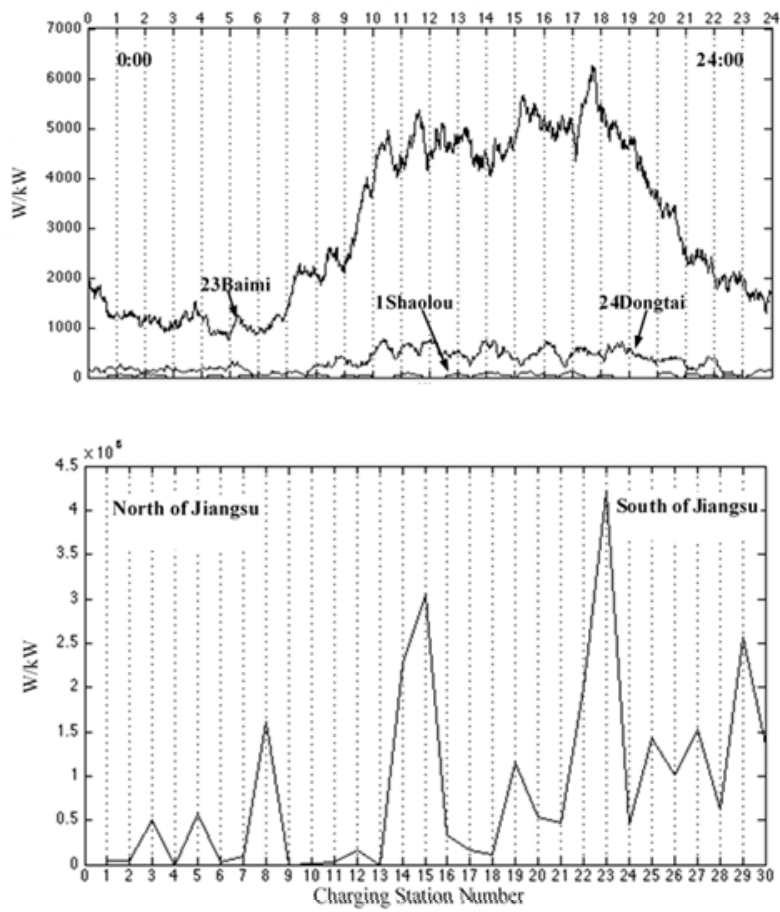

Fig.6 simulation results

The results indicates that totally, there are two picks of load during a day. Methods should be applied to order the charging time of EVs. Also, huge variation is observed in the profile of charging station-W, which indicates that the locating of charging stations should be improved.

\section{The impact of EV Load on the grid}

If not been ordered, the charging time of electric vehicle is relatively concentrated, which will impact a lot on the distribution power grid on voltage loss, harmonic and the balance of three phase.

In this passage, we consider voltage loss as the mainly impact. The loads simulated is added into the network and calculate the flow.

We classified the statistic of load during a day into 6 classes: 0:00-4:00, 4:00-8:00, 8:00-12:00, $12: 00-16: 00,16: 00-18: 00$, and 18:00-24:00. And calculate the voltage loss separately. MatPower is applied to compute the power flow, in which IEEE 30 is chosen with 27 PQ nodes. The units should be converted by: 
$W_{D}=W_{D D} / 4000$

By which $k W h$ is converted into $M w$. Firstly, we judge the impact by loss. The results are showed in Table.2.

Table.2 Loss of the grid

\begin{tabular}{ccccccc}
\hline Loss & $\mathbf{0 : 0 0 - 4 : 0 0}$ & $\mathbf{4 : 0 0 - 8 : 0 0}$ & $\mathbf{8 : 0 0 - 1 2 : 0 0}$ & $\mathbf{1 2 : 0 0 - 1 6 : 0 0}$ & $\mathbf{1 6 : 0 0 - 2 0 : 0 0}$ & $\mathbf{2 0 : 0 0 - 2 4 : 0 0}$ \\
\hline Before(P/Mw) & 67.06 & 67.06 & 67.06 & 67.06 & 67.06 & 67.06 \\
After(P/Mw) & 68.84 & 70.73 & 89.72 & 106.26 & 110.51 & 78.16 \\
Before(Q/MVAw) & 1322.56 & 1322.56 & 1322.56 & 1322.56 & 1322.56 & 1322.56 \\
After(Q/MVAw) & 1426.48 & 1532.57 & 2337.52 & 3021.99 & 3165.33 & 1877.23 \\
\hline
\end{tabular}

Variation on voltage is described by voltage deviation.

$U \%=\frac{U_{1}-U_{N}}{U_{N}} \times 100 \%$

Variation on voltage of each nodes in different periods is showed as Fig.7:
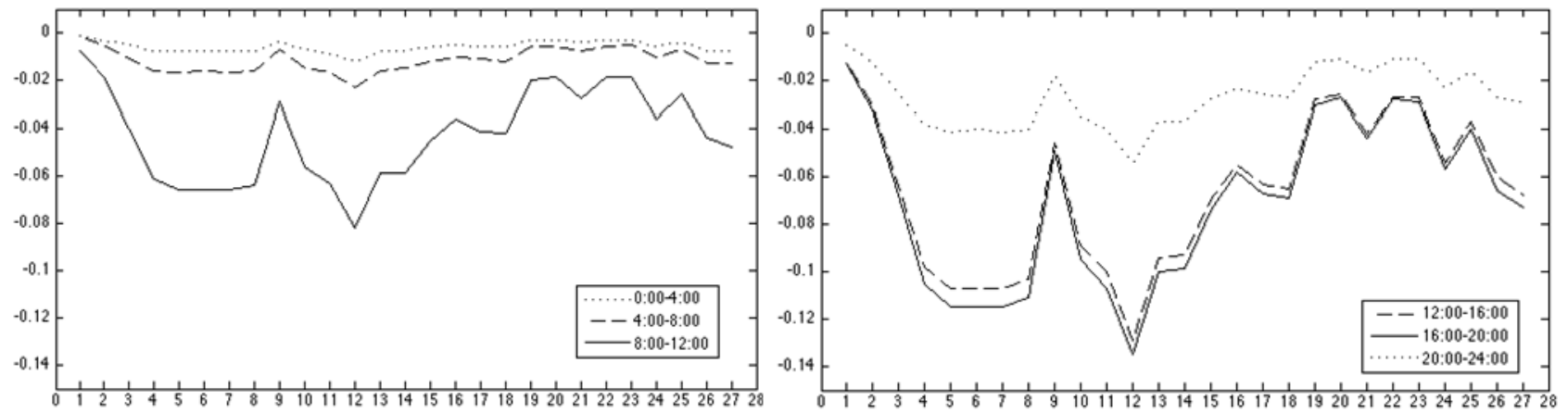

Fig.7 voltage deviation

The largest deviation is observed during 16:00-20:00. As a result, to avoid the harm of voltage deviation on the grid, methods should be taken to hold the voltage. Vehicle to grid (V2G) method has been developed recently, which treat the battery of EVs as methods to hold the frequency. Distributed power like wind and solar energy in households is also helpful of clipping the picks. Also, the degree of voltage deviation varies from charging stations, which means that variable methods should be considered in different areas.

\section{Conclusion}

The method of predicting load of EVs on highway is approximately accurate, and the impact on power grid is calculated in this passage. It suggest that the load of EVs varies from time and area, so that diverse methods should be taken in different areas and different time. Actually, there still exists some problems in the method: Firstly, due to the routine is determined by shortest path, some roads observe no cars. Secondly, queuing time and waiting time during the charging process is neglected, which can increase the accuracy of simulation. Thirdly, the result of Monte Carlo method differs in different simulation, judgements should be established to consider which the most accurate simulation result is.

\section{Acknowledgments}

This work was supported by State Grid Corporation of China. 


\section{References}

[1]GAO Ciwei and ZHANG Liang. A survey of influence of electric vehicles charging on power grid [J]. Power System Technology, 2011, 32 (2): 127-131.

[2] ZHANG Hongcai, HU Zechun, SONG Yonghua and XU Zhiwei. EV charging load prediction considering the spatial and temporal distribution [J]. Automation of Electric Power Systems, 2014, 01: 13-20.

[3] TIAN Liting, SHI Shuanglong and JIA Zhuo. A statistical model for charging power demand of electric vehicles [J]. Power System technology, 2010, 34 (11): 126-130.

[4] LUO Zhuowei, HU Zechun and SONG Yonghua. A study on plug-in electric vehicles charging load calculating [J]. Automation of Electric Power Systems, 2011, 35 (14): 36-42.

[5] Ashtari, A.; Bibeau, E.; Shahidinejad, S.; Molinski, T., PEV Charging Profile Prediction and Analysis Based on Vehicle Usage Data, in Smart Grid, IEEE Transactions on , vol.3, no.1, pp.341-350, March 2012.

[6] Galus, Matthias D.; Waraich, R.A.; Noembrini, F.; Steurs, K.; Georges, G.; Boulouchos, K.; Axhausen, K.W.; Andersson, G., Integrating Power Systems, Transport Systems and Vehicle Technology for Electric Mobility Impact Assessment and Efficient Control, in Smart Grid, IEEE Transactions on , vol.3, no.2, pp.934-949, June 2012.

[7] Tae-Kyung Lee; Bareket, Z.; Gordon, T.; Filipi, Z.S., Stochastic Modeling for Studies of Real-World PHEV Usage: Driving Schedule and Daily Temporal Distributions, in Vehicular Technology, IEEE Transactions on, vol.61, no.4, pp.1493-1502, May 2012. 